\title{
1/3 magnetization plateau and frustrated ferrimagnetism in a sodium iron phosphite
}

A.N. Vasiliev, ${ }^{1,2,3}$ O.S. Volkova, ${ }^{1,2,3}$ E.A. Zvereva, ${ }^{1}$ E.A. Ovchenkov, ${ }^{1}$ I. Munaò ${ }^{4}$ L. Clark, ${ }^{4}$ P. Lightfoot, ${ }^{4}$ E.L. Vavilova, ${ }^{5,6}$ S. Kamusella, ${ }^{6}$ H.-H. Klauss, ${ }^{6}$ J. Werner, ${ }^{7}$ C. Koo, ${ }^{7}$ R. Klingeler, ${ }^{7}$ and A.A. Tsirlin ${ }^{8}$

${ }^{1}$ Physics Faculty, Lomonosov Moscow State University, 119991 Moscow, Russia

${ }^{2}$ National University of Science and Technology "MISiS", 119049 Moscow, Russia

${ }^{3}$ Institute of Physics and Technology, Ural Federal University, 620002 Ekaterinburg, Russia

${ }^{4}$ School of Chemistry, University of St Andrews, Fife KY16 9ST, UK

${ }^{5}$ Zavoisky Institute of Physics and Technology, RAS, Kazan 420029, Russia

${ }^{6}$ Institute of Solid State Physics, Dresden Technical University, D-01069 Dresden, Germany

${ }^{7}$ Kirchhoff Institute of Physics, Heidelberg University, D-69120 Heidelberg, Germany

${ }^{8}$ Experimental Physics VI, Center for Electronic Correlations and Magnetism,

Institute of Physics, University of Augsburg, D-86135 Augsburg, Germany

The sodium-iron phosphite $\mathrm{NaFe}_{3}\left(\mathrm{HPO}_{3}\right)_{2}\left(\mathrm{H}_{2} \mathrm{PO}_{3}\right)_{6}$ is studied by ac-magnetic susceptibility, pulsed-field magnetization, specific heat, and high-frequency electron spin resonance (HF-ESR) measurements combined with Mössbauer spectroscopy and density-functional calculations. We show that this compound develops ferrimagnetic order below $T_{C}=9.5 \mathrm{~K}$ and reveals a magnetization plateau at $1 / 3$ saturation. The plateau extends to $B_{\mathrm{c}} \sim 8 \mathrm{~T}$, whereas above $B_{c}$ the magnetization increases linearly until reaching saturation at $B_{s} \sim 27 \mathrm{~T}$. The Mössbauer spectroscopy reveals two magnetically non-equivalent iron sites with the 2:1 ratio. The HF-ESR spectra are consistent with a two-sublattice ferrimagnet and additionally pinpoint weak magnetic anisotropy as well as short-range spin order that persists well above $T_{C}$. The ferrimagnetic order in the title compound is stabilized by a network of purely antiferromagnetic exchange interactions. The spin lattice comprises layers coinciding with the crystallographic (10-1) planes, with stronger couplings $J_{i} \sim 1.5 \mathrm{~K}$ within the layers and weaker couplings $J_{i}=0.3-0.4 \mathrm{~K}$ between the layers. Both intralayer and interlayer couplings are frustrated. The ensuing ferrimagnetic order arises from a subtle interplay of the frustrated but nonequivalent exchange couplings.

\section{Introduction}

The magnetization of conventional isotropic antiferromagnets increases monotonically in an applied magnetic field. Fewer systems will, however, show a more complex magnetization process with flat or nearly flat regions of the magnetization curve that are known as magnetization plateaus [1]. These plateaus will normally appear at integer fractions of the total (saturation) magnetization and manifest ferrimagnetic order induced by the magnetic field. The stabilization of ferrimagnetic phases is strongly linked to the presence of magnetic frustration. For example, triangular antiferromagnets reveal a 1/3-plateau that is most pronounced in the quantum spin-1/2 regime, where the collinear ferrimagnetic phase is stabilized by quantum fluctuations [2-4]. It does, however, persist in the classical regime as well, thanks to the stabilization by thermal fluctuations [5,6], as in the extensively studied spin-5/2 triangular multiferroic $\mathrm{RbFe}\left(\mathrm{MoO}_{4}\right)_{2}$ [7-9]. On the other hand, even classical spin systems can show magnetization plateaus of putatively quantum nature, such as the 1/3-plateau recently observed in the spin-trimer compound $\mathrm{SrMn}_{3} \mathrm{P}_{4} \mathrm{O}_{12}$ [10-13].

In the following we report a detailed investigation of a novel iron fluorophosphite showing a pronounced magnetization plateau at 1/3-saturation. The true composition, which incorporates a very small amount of fluorine, has been discussed previously (14) but for simplicity in the present work we use an idealized composition $\mathrm{NaFe}_{3}\left(\mathrm{HPO}_{3}\right)_{2}\left(\mathrm{H}_{2} \mathrm{PO}_{3}\right)_{6}$, 
abbreviated NaFP. This approximation has no bearing on the interpretation of physical properties in the present work.

The crystal structure of NaFP [14] comprises two nonequivalent positions of $S=5 / 2 \mathrm{Fe}^{3+}$ ions, both octahedrally coordinated. These $\mathrm{FeO}_{6}$ octahedra are linked into a three-dimensional network via tetrahedral phosphite groups. We conclude that the system features a ferrimagnetic ground state that underlies the formation of the broad 1/3-plateau observed in our experiments. While the ferrimagnetic ground state may look like a natural consequence of the two nonequivalent $\mathrm{Fe}$-sublattices with the $2: 1$ ratio of $\mathrm{Fe}^{3+}$ ions, individual magnetic interactions driving the formation of this ferrimagnetic state are far from being trivial. In fact, there are no direct $\mathrm{Fe}-$ $\mathrm{O}-\mathrm{Fe}$ links between the $\mathrm{FeO}_{6}$ octahedra in the structure, and there are no distinguishable sublattices in the sense of stronger ferromagnetic couplings within each sublattice and weaker antiferromagnetic couplings between the sublattices, as standard theory of ferrimagnetism would assume. Instead, we find a quasi-two-dimensional spin lattice that manifests itself in the nearly linear temperature dependence of the specific heat below $T_{C}$. We also show that the ferrimagnetic order hinges upon a very subtle interplay of frustrated exchange couplings both within the layers and between the layers. Minor variations of these couplings are supposed to lead to a qualitatively different magnetic ground state.

\section{Methods}

The sodium-iron phosphite $\mathrm{NaFP}$ was synthesized by a reaction of $\mathrm{NaF}, \mathrm{Fe}_{2} \mathrm{O}_{3}$ and $\mathrm{H}_{3} \mathrm{PO}_{3}$. This mixture was sealed in a Teflon-lined stainless steel autoclave, heated to $140{ }^{\circ} \mathrm{C}$ for 3 days, and cooled to room temperature. Pink air-stable prismatic crystals were recovered by filtration. The material was found to adopt a triclinic structure in the centrosymmetric space group, P-1, with crystal lattice parameters $a=7.5302 \AA, b=9.1696 \AA, c=9.4732 \AA, \alpha=$ $118.063^{\circ}, \beta=101.274^{\circ}, \gamma=101.192^{\circ}$ [14]. The experimental pattern obtained by the single crystal X-ray measurement fits well with the experimental powder pattern, revealing that the synthesized sample has a good level of purity.

Thermodynamic properties of NaFP were studied by measurements of ac-magnetic susceptibility $\chi(10 \mathrm{G}, 10 \mathrm{kHz}), d c$-magnetization $M$ and specific heat $C_{p}$ in the temperature range $T=2 \div 300 \mathrm{~K}$ using various options of the "Quantum Design" Physical Properties Measurements System PPMS-9T. The pulsed magnetic field measurements were performed using the home-made experimental setup with the magnetic pulse duration of about $8 \mathrm{~ms}$.

High-frequency electron spin resonance (HF-ESR) measurements were performed on fixed as well as on loose powder samples in the frequency range of $f=40-420 \mathrm{GHz}$, and in the temperature range from $2 \mathrm{~K}$ to $250 \mathrm{~K}$. A millimeter vector network analyzer was used as a source and detector of stable microwaves, and a superconducting magnet was applied to provide a magnetic field up to $15 \mathrm{~T}$ [15].

Mössbauer spectroscopy experiments were made with a commercial Wissel setup, using a ${ }^{57} \mathrm{Co} / \mathrm{Rh}$ source. The drive was run in sinusoidal mode minimizing the velocity error. The measurements were carried out in the temperature range from $2 \mathrm{~K}$ to $315 \mathrm{~K}$. The spectra were analyzed using Moessfit package by means of transmission integral fits [16].

Magnetic exchange couplings were obtained from total energies of collinear spin configurations calculated using the VASP [17] code within the DFT $+U$ formalism, where correlation effects in the $\mathrm{Fe} 3 d$ shell were included on the mean-field level. Individual exchange couplings $J_{i j}$ were defined by the spin Hamiltonian $H=\Sigma_{i j} J_{i j} \mathbf{S}_{i} \mathbf{S}_{j}$, where the summation is over bonds $i j$, and the local spin is $S=5 / 2$. The Perdew-Burke-Ernzerhof flavor of the exchangecorrelation potential [18] was applied, and several two-fold supercells were considered in order to distinguish between individual magnetic couplings in the $\mathrm{NaFe}_{3}\left(\mathrm{HPO}_{3}\right)_{2}\left(\mathrm{H}_{2} \mathrm{PO}_{3}\right)_{6}$ structure. The couplings calculated in different supercells matched within $0.1 \mathrm{~K}$, thus confirming the high accuracy of our calculation and the absence of any noticeable long-range couplings not included in the magnetic model presented below. Correlation effects were described by an effective onsite Coulomb repulsion $U_{d}=7 \mathrm{eV}$ and Hund's exchange $J_{d}=1 \mathrm{eV}$ [19]. Thermodynamic 
properties were obtained from classical Monte-Carlo simulations on finite $L \times L \times L$ lattices with periodic boundary conditions and $L \leq 8$. The simulations were performed using the "spinmc" algorithm of the ALPS simulation package [20].

\section{Thermodynamic properties}

The temperature dependences of both $a c$-magnetic susceptibility $\chi_{a c}$ and specific heat $C_{p}$, shown in Fig. 1, reveal the formation of a magnetically ordered state in NaFP below $T_{C}=9.5 \mathrm{~K}$. In a wide temperature range down to below $40 \mathrm{~K}$, the magnetic susceptibility follows the CurieWeiss law augmented by a temperature-independent term $\chi_{a c}=\chi_{0}+C /(T-\Theta)$ with $\chi_{0} \sim-5 \times 10^{-4}$ $\mathrm{emu} / \mathrm{mol}$, Curie constant $C=12.6 \mathrm{emu} \mathrm{K} / \mathrm{mol}$ and Weiss temperature $\Theta=-22 \mathrm{~K}$. The lowtemperature peak in the real part $\chi^{\prime}$ of the $a c$-susceptibility, i.e. Hopkinson maximum, is the signature of spontaneous magnetization in NaFP at $T<T_{C}$. The value of the Curie constant $C=$ $n g^{2} S(S+1) \mu_{B}^{2} N_{A} / 3 k_{B}$, where $\mu_{B}, N_{A}, k_{B}$ are Bohr, Avogadro and Boltzmann constants, respectively, is consistent with the $g$-factor $g \approx 2$ for $n=3 \mathrm{Fe}^{3+}$ ions per formula unit in the $S=$ $5 / 2$ high-spin state. The negative value of the Weiss temperature $\Theta$ signals the predominance of antiferromagnetic exchange interactions in this system.

At room temperature, the specific heat $C_{p}$ is far from the thermodynamic limit $3 R n=$ 1247.5 J/mol K. To estimate the lattice contribution to $C_{p}$ at low temperatures, we used the Debye temperature $T_{D} \approx 460 \mathrm{~K}$ extracted from the temperature dependence of the isomer shift measured by Mössbauer spectroscopy (see Supplementary Material). The lattice contribution is negligibly small at $T<T_{C}$ as shown by the dashed line in the inset to the lower panel of Fig. 1. At $T_{C}=9.5 \mathrm{~K}$, the specific heat $C_{p}$ demonstrates a $\lambda$-type anomaly. Below $T_{C}$, a linear trend $C_{p} \sim$ $T$ is observed which resembles the behavior of quasi-two-dimensional ferromagnets [21].

The field dependences of the magnetization $M$ measured in static magnetic field up to $B=$ $9 \mathrm{~T}$ and in pulsed magnetic field up to $B \sim 30 \mathrm{~T}$ measured at $T=2 \mathrm{~K}(\sim 2.4 \mathrm{~K}$ in case of pulsedfield measurements) are shown in Fig. 2. The effects of sample heating were detected through the hysteresis between the up- and down-sweeps. The magnetization rapidly reaches the plateau value of $M_{s} / 3 \sim 4.3 \mu_{\mathrm{B}}$ which persists to $B_{c} \sim 8 \mathrm{~T}$. The saturation $M_{s} \sim 13 \mu_{\mathrm{B}}$ is reached at $B_{s} \sim 27$ $\mathrm{T}$. Within these limits, the field dependence of the magnetization is mostly linear.

The application of an external magnetic field drastically changes the appearance of the $C_{p}$ vs. $T$ curves. Instead of the sharp peak at $T_{C}$, a rather broad anomaly appears signaling the redistribution of magnetic entropy over a wide temperature range. The data taken at $B=3,6$ and $9 \mathrm{~T}$ are basically very similar, as shown in the inset to Fig. 2, reflecting the persistence of the magnetic state of NaFP at the plateau. Evidently, an external magnetic field smears the transition to the magnetically ordered state out, as could be expected for ferri- or ferromagnetic systems.

\section{HF-ESR spectroscopy}

Representative HF-ESR spectra of NaFP obtained at $f \approx 230 \mathrm{GHz}$ are shown in the inset to Fig. 3. For the fixed powder, i.e. randomly-oriented material, there is a broad resonance feature extending from 7.7 to $8.4 \mathrm{~T}$, with increasing spectral weight at higher magnetic fields. The spectra drastically change in the case of loose powder, which indicates at least partial alignment of the crystallites exposed to the external magnetic field. Such behavior implies the presence of a local anisotropy. Comparison of the shape of the resonance features allows qualitative conclusions on the magnetic anisotropy, as spectral weight is shifted to the lowfield/low spectral weight regime of the randomly oriented powder. Such a behavior suggests an axial type anisotropy [22].

In the following, we will use a plain two-sublattice model to describe ferrimagnetic resonance in NaFP. This model is justified by the relative simplicity of the spectra, even though the underlying microscopic magnetic model involves as many as six Fe sublattices. The effective two-sublattice model entails two inequivalent spins $S_{1}=2 \times 5 / 2=5$ and $S_{2}=5 / 2$, which are coupled by an effective antiferromagnetic interaction $J_{\text {eff. }}$. In the classical mean field approach for 
ferrimagnetic resonances [23], the exchange interaction between the different magnetic sites is considered by the mean-field parameter $\lambda$ yielding the free energy:

$F=-B \cdot M_{1}-B \cdot M_{2}-H_{1 A} \cdot M_{1}-H_{2 A} \cdot M_{2}+\lambda M_{1} \cdot M_{2}$

where $M_{1}$ and $M_{2}$ are the two sublattice magnetizations, and $H_{1 A}$ and $H_{2 A}$ are the magnetic anisotropy fields of the sublattices. Assuming the larger sublattice magnetization $M_{l}$ pointing in the magnetic field direction and $M_{2}$ pointing oppositely, the resonance frequencies of the main ferrimagnetic excitation branches read

$\omega_{\|}^{\mathrm{I}}=\gamma_{\mathrm{eff}} \|\left(\mathrm{B}+\mathrm{H}_{\mathrm{A} \|}\right)$

$\omega^{\mathrm{I}} \perp=\gamma_{\mathrm{eff}} \perp\left(\mathrm{B}+\mathrm{H}_{\mathrm{A}^{\perp}}\right)$

$\omega_{\| \mid}^{\mathrm{I}}$ and $\omega_{\perp}^{\mathrm{I}}$ are the ferrimagnetic resonance modes with the anisotropy parallel and perpendicular to the external magnetic field, i.e. $B \| H_{i A}$ and $B \perp H_{i A}$, respectively. The two ferrimagnetic exchange resonance modes $\omega^{\mathrm{II}}$ of the two-sublattice model considered here appear at higher energies as they exhibit a zero-field gap $\Delta \sim \lambda\left(\gamma_{2} M_{1}-\gamma_{1} M_{2}\right)$. As will be discussed below, we assume $\Delta$ being larger than the frequencies used in the experiments.

The effective anisotropy field is associated to the sublattice magnetizations and anisotropic fields by

$H_{A \| \mid}=\left(H_{1 A} M_{1}+H_{2 A} M_{2}\right) /\left(M_{1}-M_{2}\right)$

$H_{A \perp}=2^{1 / 2}\left(H_{1 A} M_{1}-H_{2 A} M_{2}\right) /\left(M_{2}-M_{1}\right)$

The effective gyromagnetic ratio $\gamma_{\text {eff }}$ for both directions

$\gamma_{\text {eff } \|, \perp}=\left(M_{1}-M_{2}\right) /\left(M_{1} /\left(g_{11, \perp} \mu_{B}\right)-M_{2} /\left(g_{2 \| l, \perp} \mu_{B}\right)\right)$

is associated with the respective anisotropic g-values $\mathrm{g}_{\mathrm{i}}$. The ferrimagnetic resonance model applied here is supposed to approximate the antiferromagnetic alignment of the two sublattices considered where spins are parallel or antiparallel to the magnetic field [24].

The experimental data in Fig. 3 display a broad anomaly for randomly-oriented material which lower limit shows up as a sharp resonance feature in case of aligned powder. It is interpreted as the ferrimagnetic resonance line $\omega_{\|}^{\mathrm{I}}$ under the effect of local magnetic anisotropy and non-isotropic $g$-factors. The high-field edge of the resonance feature is interpreted as ferrimagnetic resonance mode, too, in the case where the anisotropy is orthogonal to the external magnetic field, i.e. $\omega_{\perp}^{\mathrm{I}}$. Simulation of the data by means of Eq. (1) and Eq. (2) yields $\mathrm{g}_{\|}=1.97, g_{\perp}$ $=2.07$, and $H_{1 \mathrm{~A}}=2 \cdot H_{2 \mathrm{~A}}=0.28 \mathrm{~T}$. Applying Eqs. (3) and (4) with these simulation parameters yields the effective anisotropic fields $\mathrm{H}_{\mathrm{A} \|}=0.7 \mathrm{~T}$ and $\mathrm{H}_{\mathrm{A}_{\perp}}=-0.6 \mathrm{~T}$.

As the ferromagnetic exchange modes $\omega^{\mathrm{II}}{ }_{\|}$and $\omega^{\mathrm{II}}{ }_{+}$are not observed in the spectra, the mean-field parameter is not fixed by the HF-ESR data. However, the absence of the highfrequency mode in the accessible frequency range implies $\lambda>0.22 \mathrm{~T}^{2} / \mathrm{GHz}$. Note that the ferrimagnetic resonance model described above is not valid above $B_{c}$ as the spins in that field region are not antiparallel or parallel to the magnetic field due to the canting of the spins. However, there is no discontinuity in the experimental data around $B_{c}$ and the low-field model describes the experimental data well in the whole field range.

The temperature dependence of the HF-EPR spectra at $f \approx 230 \mathrm{GHz}$ illustrates the evolution of ferrimagnetic order upon cooling, as shown in Fig. 4. At $T=250 \mathrm{~K}$, the spectrum exhibits a single resonance associated to $g=2.003(5)$. Upon cooling below $\sim 125 \mathrm{~K}$, the resonance line inhomogeneously broadens and two anomalies are resolved below about $20 \mathrm{~K}$. The evolution of the resonance features is particularly evident if $B_{\text {res } 1}$ in Fig. 4 is considered. There is a pronounced shift at around $10 \mathrm{~K}$, which signals the formation of local magnetic fields in the long-range ordered ferrimagnetic phase. In addition, the shift of resonance fields at higher temperature implies the evolution of internal magnetic fields well above $T_{C}$. As seen in the inset of Fig. 4, there are small changes of spectral weight and broadening of the resonance line extending up to $125 \mathrm{~K}$. These observations suggest the presence of internal magnetic field in the paramagnetic phase and hence the evolution of short-ranged magnetic order in this temperature regime. 


\section{Mössbauer spectroscopy}

Zero-field Mössbauer spectra in NaFP consist of a single doublet down to $T_{C}=9.5 \mathrm{~K}$. Below $T_{C}$, it splits into two distinct magnetic sextets, as shown in Fig. 5, indicating the presence of two main nonequivalent iron sites. Both iron sites have indistinguishable isomer shifts $(\delta)$ and electric field gradient values (EFG). Consequently these parameters were assumed equal for both sites. The room-temperature isomer shift of $\delta=0.42 \mathrm{~mm} / \mathrm{s}$ is typical for octahedrally coordinated high-spin $\mathrm{Fe}^{3+}$ ions $(S=5 / 2)$ which is also supported by the observed large magnetic hyperfine field $B_{\text {hyp }}>50 \mathrm{~T}$ [25]. The negligible orbital contribution to the EFG in the high spin state is in line with the small quadrupole splitting of about $0.17 \mathrm{~mm} / \mathrm{s}$.

Below $T_{C}=9.5 \mathrm{~K}$, the spectra split into two well distinguishable sextets showing different temperature dependencies of the magnetic order parameter $B_{\text {hyp }}$, as shown in Fig. 6a. A signal ratio of 65(1):35(1) can be assigned to the crystallographic sites Fe2 and Fe1, where Fe2 is the site of the smaller magnetic hyperfine field. Both field values approach each other for low temperatures with a saturation field corresponding to 4.3(2) $\mu_{\mathrm{B}}$, assuming a dominant core contribution of $13 \mathrm{~T} / \mu_{\mathrm{B}}$ [26]. A small paramagnetic doublet with $5 \%$ signal fraction is attributed to impurities. A fit of $B_{h y p}(T)$ with a critical exponent behavior and a Gaussian distribution of $T_{C}$ for temperatures $T \geq T_{C} / 2$ yields $0.338(1)$ and $0.234(1)$ as critical exponents for site Fe 2 and Fe1, respectively. It means that the Fe2-site has typical three-dimensional behavior, whereas the dimensionality of magnetic interactions at the Fe1-site may be reduced.

To study the magnetic coupling of the two iron sites, we applied an external magnetic field transverse to the gamma beam ranging between $0 \mathrm{~T}$ and $6.3 \mathrm{~T}$. This experiment was done at $5 \mathrm{~K}$ comprising sufficient contrast between the both site's hyperfine fields and yet sufficiently static character of the moments. The results of this experiment are shown in Fig. 6b. It unambiguously proves the ferrimagnetic coupling of the spins, with $\mathrm{Fe} 2$ and Fe1 spins aligning parallel and antiparallel to the applied field, respectively.

As predicted by the Weiss mean field model of ferrimagnetism, the ordered moment, quantified by $B_{h y p}$, is expected to increase upon the application of an external field at finite temperatures. The total field measured at the Fe nucleus is the vector sum $\boldsymbol{B}_{\text {tot }}=\boldsymbol{B}_{\text {hyp }}+\boldsymbol{B}_{\text {ex }}$. For ${ }^{57} \mathrm{Fe}$ in a ferromagnet, $\boldsymbol{B}_{\text {hyp }}$ and $\boldsymbol{B}_{\boldsymbol{e x}}$ will be antiparallel to each other, so that $B_{\text {tot }}$ is expected to decrease, whereas for the spins with antiparallel orientation $\boldsymbol{B}_{h y p}$ and $\boldsymbol{B}_{\boldsymbol{e x}}$ will add constructively. For field $B_{e x}<2 \mathrm{~T}$, the hyperfine field $B_{h y p}$ increases faster than $B_{e x}$, because the total ferrimagnetic moment is oriented along the field. Once aligned, the ferro- and antiferromagnetic characters of the single moments manifest themselves by the reduction and continuous increase of the total field, respectively. Above $5 \mathrm{~T}$, even the antiferromagnetically coupled Fe1 moments are forced progressively in the field direction. Consequently, the increase in the total field is slowed down. This corresponds to approaching the exchange field and thus the end of the $1 / 3$ magnetization plateau.

We fitted the total fields phenomenologically using the following formula:

$$
B_{t o t}=B_{s}\left(1-f^{*} \exp \left(-a^{*} B_{e x} / k_{B} T\right)\right) \pm B_{e x}
$$

obtaining $f=0.18(1)$, in good agreement with the Weiss model $(0.16)$ for $J=5$, and $a=0.15(1)$ $\mathrm{meV} / \mathrm{T}$ corresponding to $2.7 \mu_{\mathrm{B}}$. The latter value is an effective value describing not only thermal fluctuations but crystal anisotropy as well. Therefore, it is lower than the saturated magnetization. Anisotropy and further details of the analysis are discussed in the Supplementary Material.

\section{First-principles calculations}

Prior to calculating magnetic exchange couplings, we relaxed hydrogen positions in the experimental crystal structure, because these positions are usually determined with insufficient accuracy, owing to the weak x-ray scattering from hydrogen atoms. Indeed, the structure optimization resulted in realistic $\mathrm{O}-\mathrm{H}$ distances of $1.0 \AA$ in contrast to $0.73-0.78 \AA$ reported experimentally [14]. Likewise, the uniform $\mathrm{P}-\mathrm{H}$ distances of $1.40 \AA$ in the $\mathrm{HPO}_{3}$ groups were found, whereas the minor substitution of $\mathrm{H}$ atoms by $\mathrm{F}$ was neglected in the present calculations. 
The resulting crystal structure with the optimized positions of hydrogen, as given in the Supplementary Material, was used in all subsequent calculations.

Isotropic exchange couplings $\left(J_{i}\right)$ in $\mathrm{NaFP}$ are listed in Table I. By comparing exchange couplings calculated in different supercells, we are able to exclude any long-range exchanges beyond the six nearest-neighbor couplings presented in this Table. All the couplings are antiferromagnetic (AFM) and involve superexchange via multiple oxygen atoms, because the $\mathrm{Fe}^{3+} \mathrm{O}_{6}$ octahedra are connected via non-magnetic phosphite groups only. The couplings do not show any clear signatures of a dimerization within the Fe2 sublattice or of a separation between the $\mathrm{Fe} 1$ and $\mathrm{Fe} 2$ sublattices. Instead, we find four stronger couplings of about $2.0 \mathrm{~K}$ and two weaker couplings on the order of $0.5 \mathrm{~K}$. The four stronger couplings form magnetic planes that coincide with the (10-1) crystallographic planes, whereas the two remaining weak couplings connect these planes into a three-dimensional lattice, as shown in Fig. 7.

The intraplane couplings $J_{2}, J_{3}$, and $J_{6}$ form triangular units. While frustrated, they do not prevent the system from long-range ordering because each side of the triangle features a different coupling. Normally, one expects that two strongest couplings on the triangle stabilize antiparallel spins, while the weakest coupling is left with the parallel spin arrangement [27], but the case of $\mathrm{NaFP}$ is different. Here, magnetic order is stabilized by $J_{3}$ and $J_{6}$ that are slightly weaker than $J_{2}$, yet twice more abundant. The resulting spin arrangement shown in Fig. 7 gives rise to ferrimagnetic order within the plane. The spins are parallel within each of the Fe1 and Fe2 sublattices, and antiparallel between the sublattices.

The interplane couplings $J_{1}$ and $J_{5}$ are also frustrated by forming triangular loops with the intraplane coupling $J_{4}$. Following the same argument, the ground-state configuration should be stabilized by the coupling $J_{5}$, which is weaker than $J_{1}$ but twice more abundant and thus provides a larger stabilization energy for the ground-state magnetic configuration. The coupling $J_{5}$ is diagonal in nature and stabilizes ferromagnetic order between the planes, so that net moments of each layer add up forming a macroscopic ferrimagnetic configuration with the total magnetization $1 / 3 M_{s}$, where $M_{s}$ is the total saturation magnetization. If, on the other hand, the interlayer order would follow the weaker coupling $J_{1}$, net moments of the neighboring layers will cancel each other and result in an overall antiferromagnetic ground state.

The ferrimagnetic order in NaFP obtained in our model is consistent with experimental observations. Our microscopic magnetic model can be further verified by a direct simulation of thermodynamic properties. In Fig. 8, we present experimental and simulated magnetic susceptibilities that coincide both at high temperatures and in the vicinity of $T_{C}$ [28]. This perfect match was achieved by rescaling all exchange couplings by $-30 \%$. A similar effect can be produced by increasing $U_{d}$ in DFT $+U$ to $8 \mathrm{eV}$, which is larger than $U_{d}=7 \mathrm{eV}$ appropriate for $\mathrm{LaFeO}_{3}$ [19]. This reflects an ambiguity in the choice of $U_{d}$ when exchange couplings are weak.

The re-scaled exchange couplings are listed in the last column of Table II. They provide a good fit of both magnetic susceptibility and magnetization isotherm, as shown in Fig. 8. We were able to reproduce not only the saturation field $B_{s} \sim 27 \mathrm{~T}$ but also the behavior below the saturation. The magnetization of NaFP reaches one third of saturation already in low fields and shows a broad plateau that extends up to $8 \mathrm{~T}$. The features of the calculated curve are slightly broader than in the experiment. This discrepancy can be attributed to magnetic anisotropy that will broaden the transitions and to the effect of sample heating in the pulsed-field measurement.

\section{Discussion and summary}

The 1/3-plateau in the magnetization is typically observed for ferrimagnetic phases stabilized by an external magnetic field. For example, $\mathrm{SrMn}_{3} \mathrm{P}_{4} \mathrm{O}_{14}$ has an incommensurate antiferromagnetic ground state [29] that is suppressed in the magnetic field of about $2 \mathrm{~T}$ giving rise to the ferrimagnetic phase on the 1/3-plateau [13]. Despite showing a very similar magnetization curve, NaFP is qualitatively different, because its ground state is ferrimagnetic already in zero field. This ferrimagnetic ground state is revealed by our $a c$ - susceptibility measurements showing typical signatures of a ferrimagnetic transition at $T_{C}=9.5 \mathrm{~K}$. 
The ferrimagnetic order also ensues from the rather intricate microscopic magnetic model that reveals several competing antiferromagnetic interactions both within the layers and between the layers. The formation of net magnetic moment within the layer is controlled by the absolute values of $J_{2}, J_{3}$, and $J_{6}$. Likewise, the $2 J_{5}>J_{1}$ regime of the interlayer couplings ensures that net magnetic moments of each layer are parallel, thus giving rise to the macroscopic ferrimagnetic configuration. On the other hand, the $2 J_{5}<J_{1}$ regime would produce an antiferromagnet. This subtle balance of different ordered states is typical for frustrated antiferromagnets and implies that an effective coupling between the layers is relatively weak $\left(2 J_{5}-J_{1} \sim 0.2 \mathrm{~K}\right)$, about 10 times weaker than the intralayer couplings, thus manifesting quasi-two-dimensional nature of the compound. Remarkably, NaFP shows linear behavior of the specific heat at low temperatures, as expected in a two-dimensional Heisenberg ferro- or ferrimagnet [21].

With the help of applied field, Mössbauer spectroscopy proves the typical behavior of ferrimagnetically coupled spin-5/2 with the 2:1 abundance. The absolute hyperfine field values are in excellent agreement with the macroscopically observed saturation moment of $4.3 \mu_{\mathrm{B}}$ in magnetization measurements.

Our HF-ESR data reinforce the ferrimagnetic ordering scenario. The ferrimagnetic resonance line reveals weak anisotropy on the order of $0.2 \mathrm{~K}$, less than $1 \%$ of the characteristic exchange energy given by the Curie-Weiss temperature $\Theta=-22 \mathrm{~K}$. This weak anisotropy is consistent with the spin-5/2 nature of $\mathrm{Fe}^{3+}$ having a very small orbital moment and the paramagnetic $g$-value close to 2.0. Interestingly, ESR data suggest that local magnetic fields persist well above $T_{C}$, which is typical for frustrated magnets, where long-range order is impeded by competing exchange couplings. Indeed, $T_{C}=9.5 \mathrm{~K}$ is well below $|\Theta|=22 \mathrm{~K}$, revealing tangible effect of the frustration on the magnetic ordering.

Finally, the field $B_{c} \sim 8 \mathrm{~T}$ marks the end of the 1/3-plateau and the transformation from the collinear ferrimagnetic state into a canted configuration where the minority Fel sublattice is no longer antiparallel to the majority Fe 2 sublattice. In NaFP, this transformation seems to be a rather smooth crossover that has no visible effect on the resonance lines. This is different from, e.g., triangular-lattice magnets, where distinct phases are observed at and above the 1/3-plateau [30], and additional resonance lines appear when magnetic field exceeds the plateau range [9].

\section{Acknowledgements}

AT thanks Johannes Richter for stimulating and insightful discussions. AT was supported by the Federal Ministry for Education and Research through the Sofja Kovalevskaya Award of Alexander von Humboldt Foundation. This work was supported in part by Russian Foundation for Basic Research grants 14-02-00111, 14-02-00245, 16-02-00021, from the Ministry of Education and Science of the Russian Federation in the framework of Increase Competitiveness Program of NUST «MISiS» (№ K2-2015-075 and № K4-2015-020) and by Act 211 of the Government of Russian Federation, agreement № 02.A03.21.0006. EAZ, JW and RK acknowledge support by the Excellence Initiative of the German Federal Government and States. PL thanks EPSRC (EP/K503162/1) for partial support of a studentship to IM and the Leverhulme Trust for the award of a post-doctoral fellowship (RPG-2013-343) to LC. SK is grateful for the funding by SSP1458 of the DFG.

\section{References}

1. O.A. Starykh, Rep. Progr. Phys. 78, 052502 (2015).

2. A.V. Chubukov and D.I. Golosov, J. Phys.: Condens. Matter 3, 69 (1991).

3. J. Alicea, A.V. Chubukov, and O.A. Starykh, Phys. Rev. Lett. 102, 137201 (2009).

4. D. Yamamoto, G. Marmorini, and I. Danshita, Phys. Rev. Lett. 114, 027201 (2015).

5. H. Kawamura and S. Miyashita, J. Phys. Soc. Jpn. 54, 4530 (1985).

6. L. Seabra, T. Momoi, P. Sindzingre, and N. Shannon, Phys. Rev. B 84, 214418 (2011).

7. L.E. Svistov, A.I. Smirnov, L.A. Prozorova, O.A. Petrenko, A. Micheler, N. Buttgen, A.Ya. Shapiro, and. L.N. Demianets, Phys. Rev. B 74, 024412 (2006). 
8. M. Kenzelmann, G. Lawes, A.B. Harris, G. Gasparovic, C. Broholm, A.P. Ramires, G.A. Jorge, M. Jaime, S. Park, Q. Huang, A.Ya. Shapiro, and L.N. Demianets, Phys. Rev. Lett. 98, 267205 (2007).

9. A.I. Smirnov, H. Yashiro, S. Kimura, M. Hagiwara, Y. Narumi, K. Kindo, A. Kikkawa, K. Katsumata, A.Ya. Shapiro, and. L.N. Demianets, Phys. Rev. B 75, 134412 (2007).

10. T. Yang, Y. Zhang, S.H. Yang, G.B. Li, M. Xiong, F.H. Liao, and J.H. Lin, Inorg. Chem. 47, 2562 (2008).

11. M. Hase, T. Yang, R.H. Cong, J.H. Lin, A. Matsuo, K. Kindo, K. Ozawa, and H. Kitazawa, Phys. Rev. B 80, 054402 (2009).

12. M. Hase, M. Matsuda, K. Kaneko, N. Metoki, K. Kakurai, T. Yang, R.H. Cong, J.H. Lin, K. Ozawa, and H. Kitazawa, Phys. Rev. B 84, 214402 (2011).

13. M. Hase, V.Y. Pomjakushin, A. Donni, T. Yang, R.H. Cong, and J.H. Lin, J. Phys. Soc. Jpn. 83, 104701 (2014).

14. I. Munaò, E.A. Zvereva, O.S. Volkova, A.N. Vasiliev, A.R. Armstrong and P. Lightfoot, Inorg. Chem. 55, 2558 (2016).

15. C. Golze, A. Alfonsov, R. Klingeler, B. Büchner, V. Kataev, C. Mennerich, H.-H. Klauss, M. Goiran, J.-M. Broto, H. Rakoto, S. Demeshko, G. Leibeling, and F. Meyer, Phys. Rev. B 73, 224403 (2006).

16. S. Kamusella and H.-H. Klauss, "Moessfit - free Mössbauer fitting program", accepted to Hyperfine Interactions (2016).

17. G. Kresse and J. Furthmuller, Comput. Mater. Sci. 6, 15 (1996); Phys. Rev. B 54, 11169 (1996).

18. J.P. Perdew, K. Burke, and M. Ernzerhof, Phys. Rev. Lett. 77, 3865 (1996).

19. These values of the correlation parameters were inferred from reference calculations for $\mathrm{LaFeO}_{3}$, where we obtained $J_{a b}=61 \mathrm{~K}$ and $J_{c}=55 \mathrm{~K}$ resulting in the Neel temperature of $734 \mathrm{~K}$ in excellent agreement with the experimental $J_{a b} \sim J_{c} \sim 56.8 \mathrm{~K}[31]$ and $T_{N}=738$ $\mathrm{K}[32]$.

20. A. Albuquerque, F. Alet, P. Corboz, et al., J. Magn. Magn. Mater. 310, 1187 (2007).

21. I. Yamada, J. Phys. Soc. Jpn. 33, 979 (1972).

22. C.P. Poole, H.A. Farach, Bull. Magn. Res. 1, 162 (1979).

23. R.K. Wangsness, Phys. Rev. 91, 1085 (1953).

24. J. S. Smart, Magnetism. 63 (1963).

25. P. Gütlich, Z. Anorg. Allg. Chem. 638, 15 (2012).

26. O. Eriksson and A. Svane, Journal of Physics: Condensed Matter 11589 (1989).

27. K. M. Ranjith, R. Nath, M. Skoulatos, L. Keller, D. Kasinathan, Y. Skourski, and A. A. Tsirlin, Phys. Rev. B 92, 094426 (2015).

28. Here, we used $g=1.98$ and a temperature-independent contribution $\chi_{0}=-0.001 \mathrm{emu} / \mathrm{mol}$ that may be ascribed to the sample holder.

29. M. Hase, V.Yu. Pomjakushin, L. Keller, A. Dönni, O. Sakai, T. Yang, R. Cong, J. Lin, K. Ozawa, and H. Kitazawa, Phys. Rev. B 84, 184435 (2011).

30. C. Griset, S. Head, J. Alicea, and O.A. Starykh, Phys. Rev. B 84, 245108 (2011).

31. R.J. McQueeney, J.-Q. Yan, S. Chang, and J. Ma, Phys. Rev. B 78, 184417 (2008).

32. D. Treves, Phys. Rev. 125, 1843 (1962).

33. K. Momma and F. Izumi, J. Appl. Crystallogr. 44, 1272 (2011). 
Table I. Exchange couplings $J_{i}$ (in $\mathrm{K}$ ) in NaFP. The relevant $\mathrm{Fe}-\mathrm{Fe}$ distances are in units of $\AA$, whereas $z_{i}$ 's denote the number of couplings per site. The last column contains $J_{i}$ 's rescaled by $-30 \%$ in order to match the experimental susceptibility and magnetization, see Fig. 8.

\begin{tabular}{|c|c|c|c|c|}
\hline & $z_{\mathrm{Fe} 1}, z_{\mathrm{Fe} 2}$ & $\mathrm{Fe}-\mathrm{Fe}$ distance $(\AA)$ & $J_{i}(\mathrm{~K})$ & $J_{i}(\mathrm{~K})$, rescaled \\
\hline$J_{1}$ & 0,1 & 4.821 & 0.6 & 0.4 \\
\hline$J_{2}$ & 0,1 & 4.943 & 2.4 & 1.6 \\
\hline$J_{3}$ & 2,1 & 5.620 & 2.2 & 1.5 \\
\hline$J_{4}$ & 1,1 & 5.788 & 2.0 & 1.3 \\
\hline$J_{5}$ & 2,2 & 5.926 & 0.4 & 0.3 \\
\hline$J_{6}$ & 2,2 & 6.317 & 2.3 & 1.5 \\
\hline
\end{tabular}

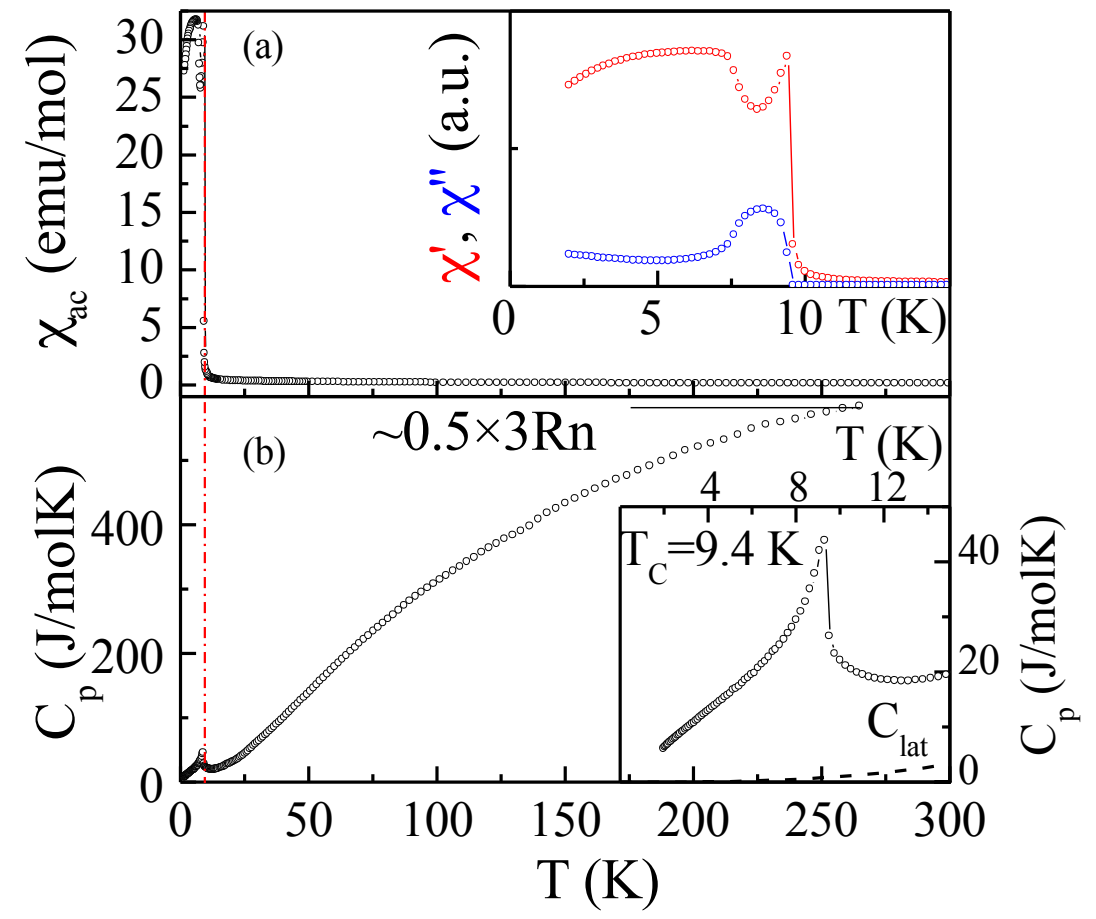

Fig. 1. a) The temperature dependences of $a c$-susceptibility in NaFP. The inset represents both real $\chi^{\prime}$ and imaginary $\chi^{\prime \prime}$ parts at low temperatures; b) the temperature dependence of the specific heat $C_{p}$. The Inset enlarges the region around $T_{C}=9.5 \mathrm{~K}$. The lattice contribution $C_{\text {lat }}$ at low temperatures is shown by the dashed line.

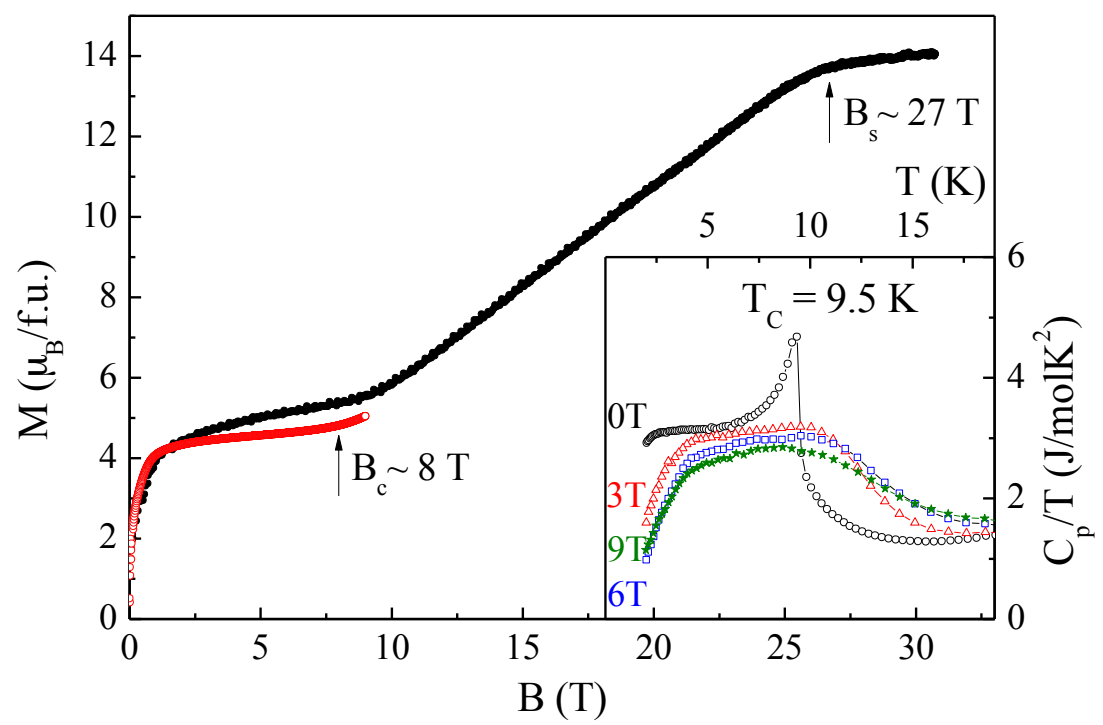


Fig. 2. The field dependences of magnetization $M$ in NaFP taken in static (open symbols) and pulsed magnetic field (filled symbols) at $T=2 \mathrm{~K}(2.4 \mathrm{~K})$. The inset shows the reduced specific heat $C_{p} / T$ taken in different magnetic fields $B=0,3,6$ and $9 \mathrm{~T}$.

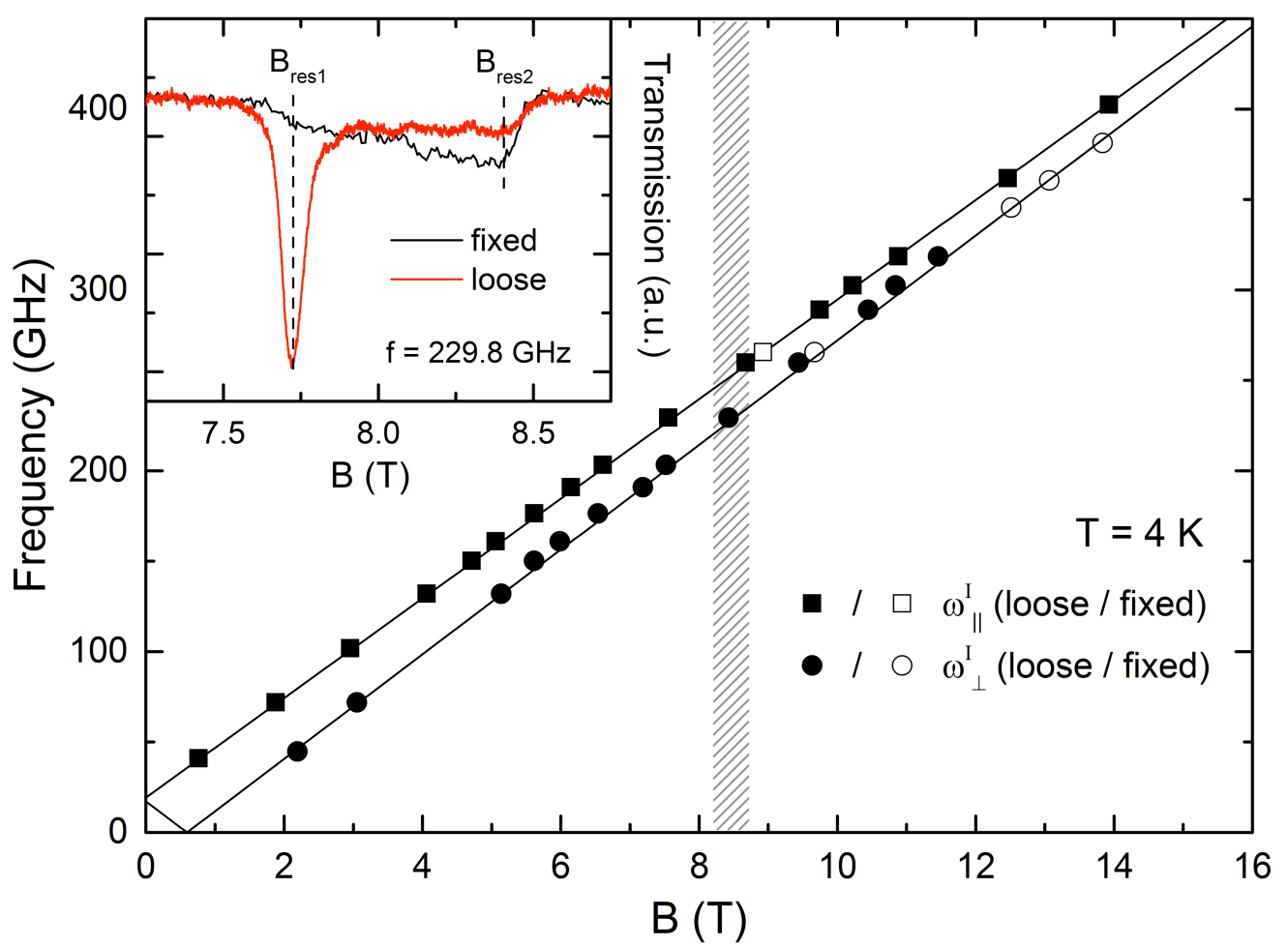

Fig. 3. Frequency-resonance field diagram, at $T=4 \mathrm{~K}$, of NaFP. The inset shows representative spectra obtained on loose and fixed powder, at $f \approx 230 \mathrm{GHz}$. Filled (open) symbols mark the resonance fields obtained on loose (fixed) powder. Lines are simulations according to Eq. (1) and Eq. (2). The shaded area indicates $\mathrm{B}_{\mathrm{c}} \approx 8 \mathrm{~T}$.

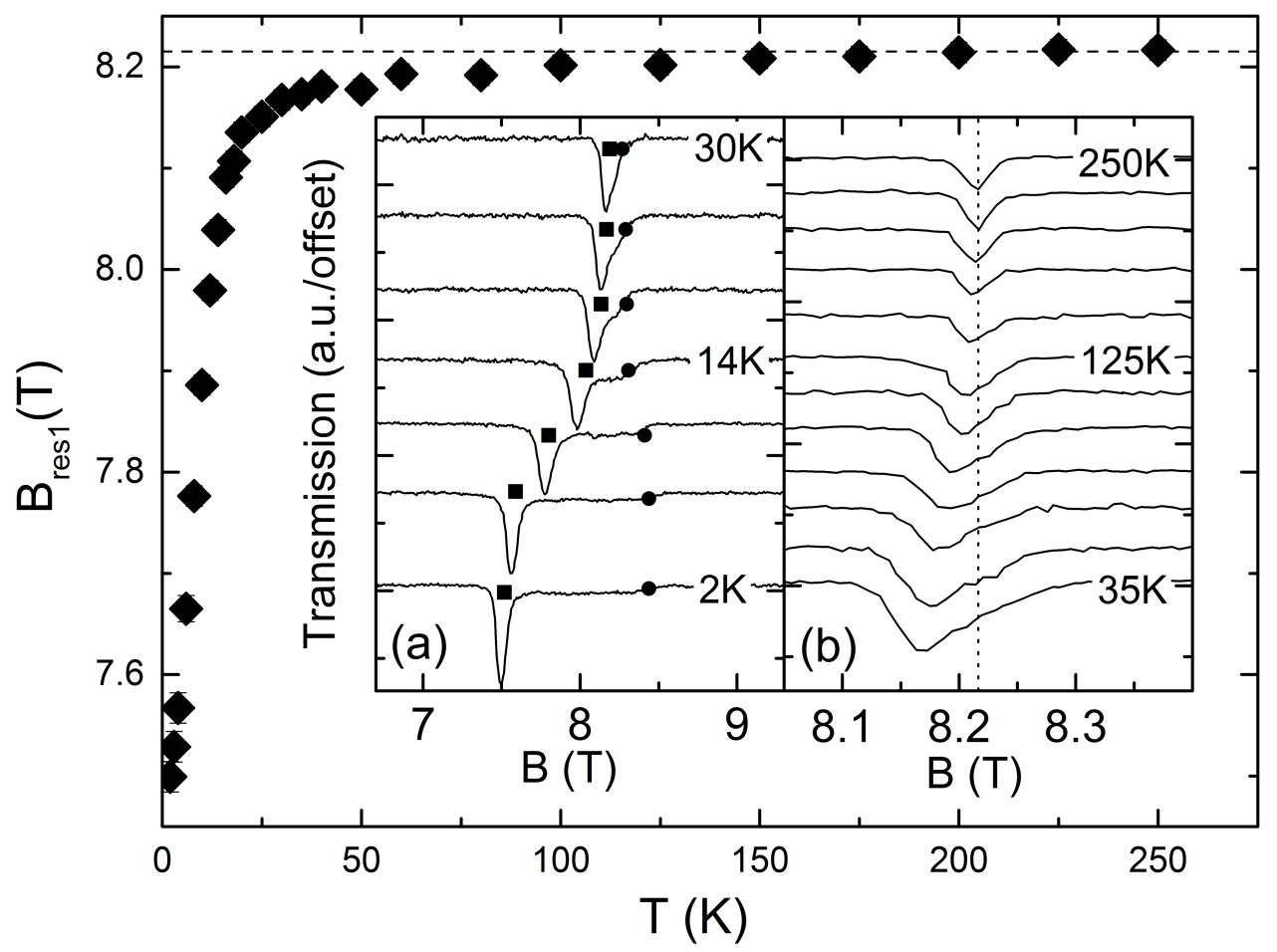


Fig. 4. Temperature dependence of the main resonance in NaFP obtained from loose powder HFESR data at $f \approx 230 \mathrm{GHz}$. The horizontal dashed line indicates the resonance field at $\mathrm{T}=250 \mathrm{~K}$. The inset highlights changes of the spectra (a) at $2 \mathrm{~K} \leq \mathrm{T} \leq 30 \mathrm{~K}$ and (b) $35 \mathrm{~K} \leq \mathrm{T} \leq 250 \mathrm{~K}$. At $\mathrm{T}$ $\approx 125 \mathrm{~K}$, a single line broadens and splits upon cooling. Squares and circles in the inset (a) indicate $\mathrm{B}_{\text {res } 1}$ and $\mathrm{B}_{\text {res } 2}$, respectively.

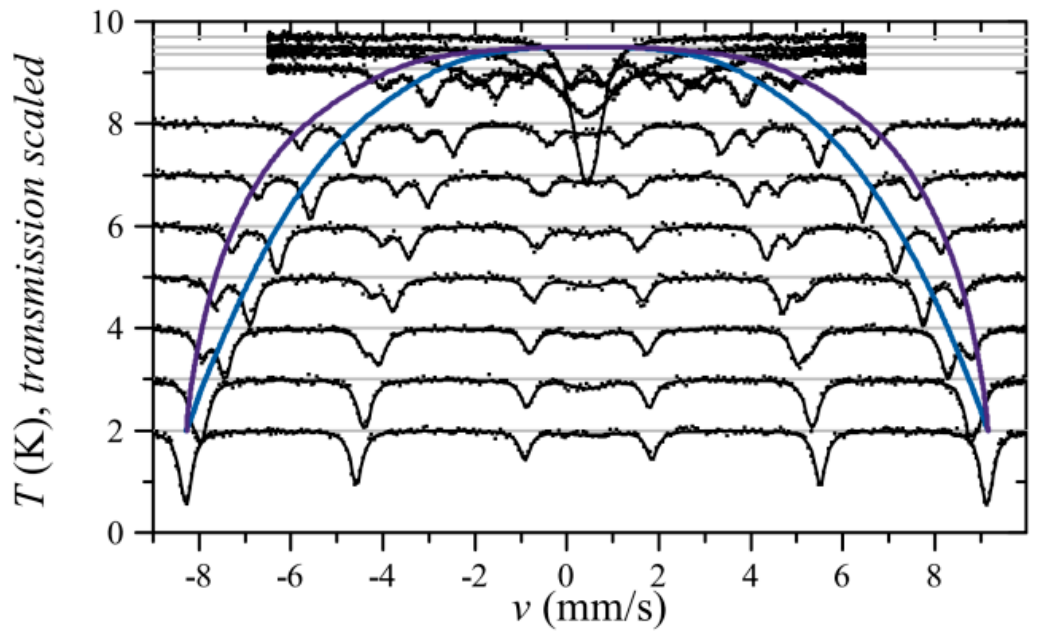

Fig. 5. The temperature evolution of zero field Mössbauer spectra in NaFP.
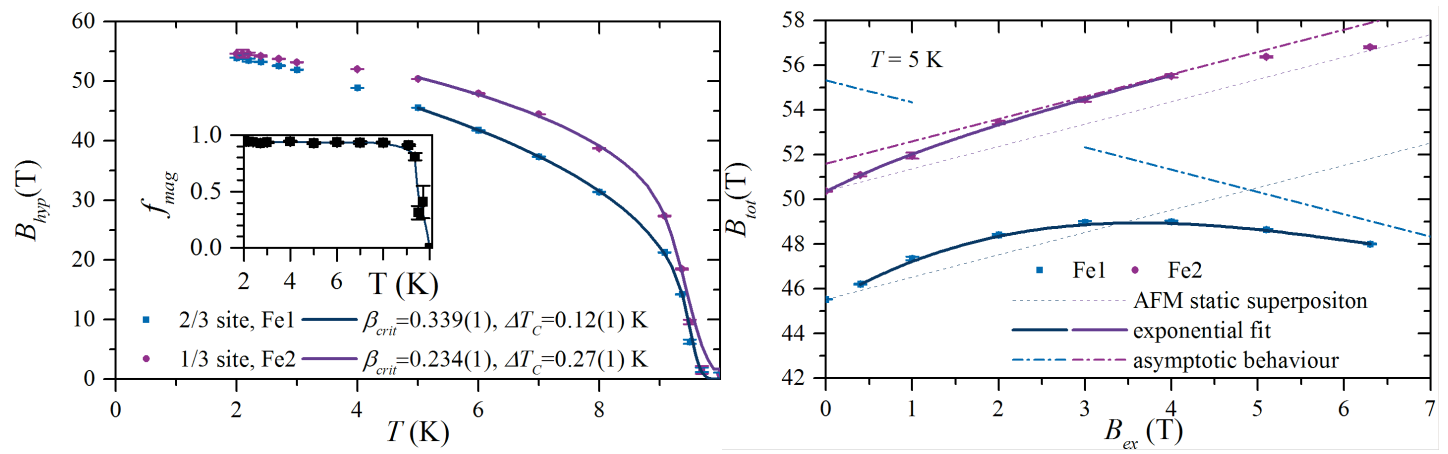

Fig. 6. a) magnetic order parameter $B_{\text {hyp }}$ and magnetically ordered fraction $f_{\text {mag }}$ b) total field $B_{\text {tot }}$ as a function of applied field $B_{e x}$, indicative of a ferrimagnetic coupling
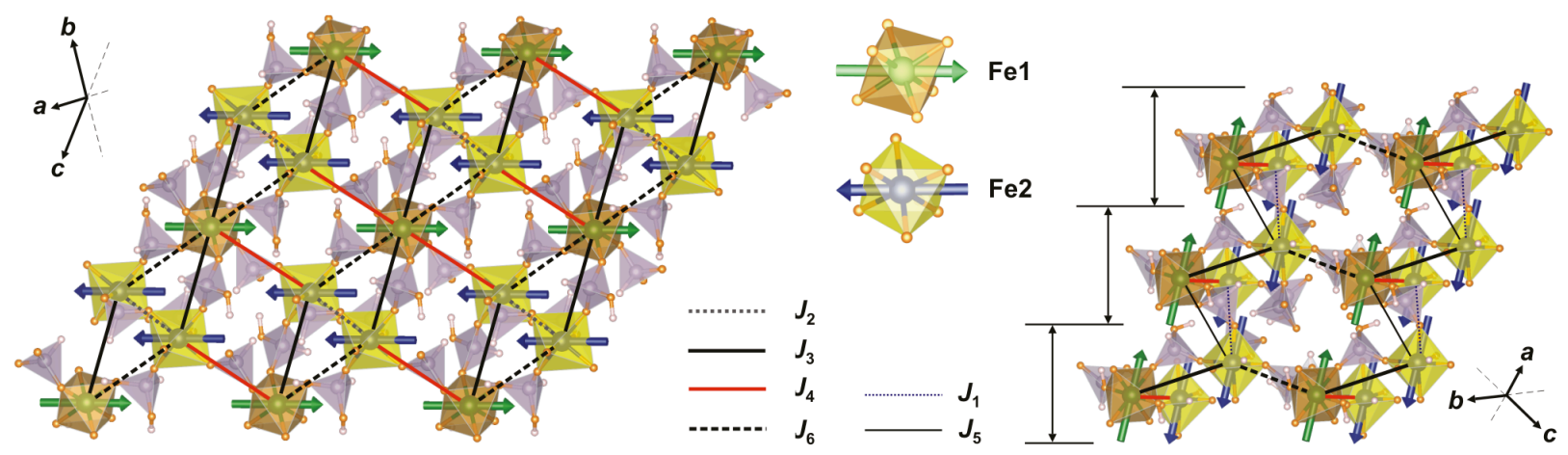

Fig. 7. Microscopic magnetic model of NaFP. a) magnetic planes formed by the four stronger couplings $J_{2}, J_{3}, J_{4}$, and $J_{6}$. b) stacking of the magnetic planes, the planes are connected by the weaker couplings $J_{1}$ and $J_{5}$. The ferrimagnetic ground state stabilized by the couplings $J_{3}-J_{6}$ is also shown. An arbitrary direction of the magnetic moments is chosen. The VESTA software [33] was used for crystal structure visualization. 

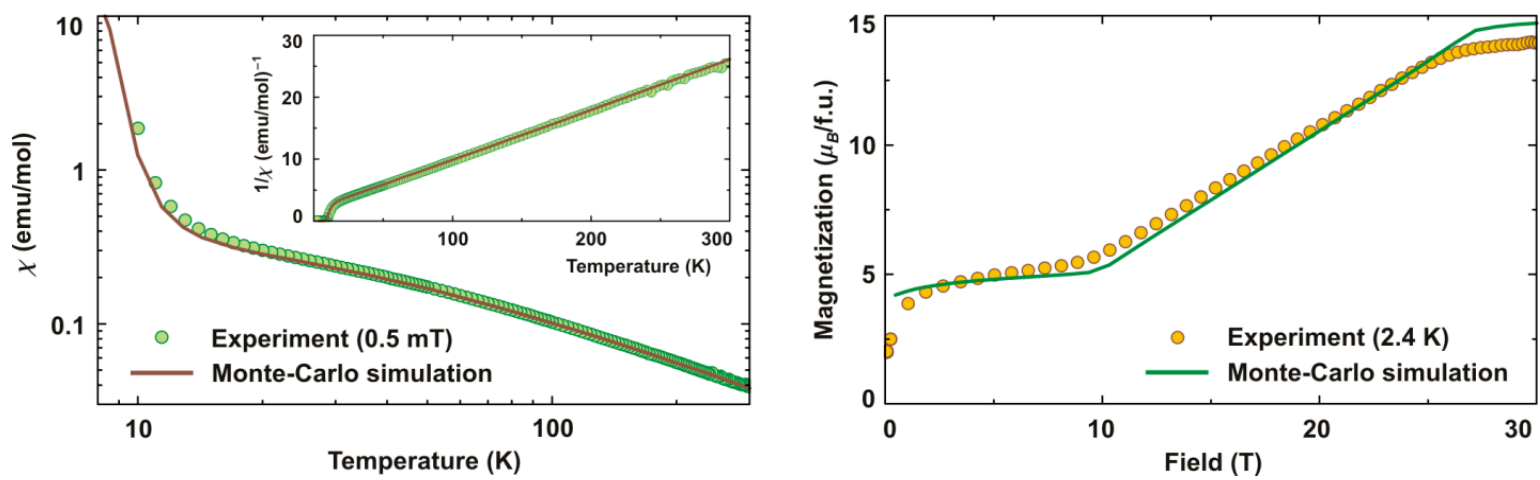

Fig. 8. a) magnetic susceptibility of NaFP compared with the classical Monte-Carlo simulation for the microscopic magnetic model with $J_{i}$ rescaled from Table I. b) the experimental and simulated magnetization curves. 